\title{
INVESTIGACIÓN-ACCIÓN PARTICIPATIVA IMPLEMENTANDO EL MÉTODO LUME: EVALUACIÓN DE UN SISTEMA FAMILIAR AGROECOLÓGICO (BELLA VISTA, CORRIENTES, ARGENTINA)
}

\section{Participatory action research to apply Lume methodology: Assessing an agroecological family farming (Bella Vista, Corrientes, Argentina)}

\author{
Pereda, María M. ${ }^{1}$; Iermanó, María J. ${ }^{2}$; Fleita, Fernando A. ${ }^{1-3}$; Almada, Carolina ${ }^{4}$; \\ Ortíz, Silvia ${ }^{3}$ \\ ${ }^{1}$ INCUPO; ${ }^{2}$ Asesora privada - LIRA, FCAyF, UNLP; ${ }^{3}$ Grupo Agroecológico Las Tres Colonias; \\ ${ }^{4}$ Departamento Alimentos, INTI NEA. E-mail: paiapereda67@yahoo.com.ar; \\ mariajoseiermano@gmail.com; fredybellavista@yahoo.com.ar; almada@inti.gob.ar
}

\begin{abstract}
RESUMEN
En el centro-oeste de la provincia de Corrientes, la expansión del modelo de agricultura convencional representó un quiebre de las producciones para autoconsumo. Sin embargo, aún persisten agricultores familiares diversificados para autoconsumo y comercialización, como las familias productoras del Grupo Agroecológico Las Tres Colonias. Estos agroecosistemas no pueden evaluarse únicamente a través de la rentabilidad o la productividad, ya que los objetivos se rigen por elementos extraeconómicos. Una propuesta adecuada para ello es el método de análisis económico-ecológico de agroecosistemas (método Lume). El objetivo del trabajo fue evaluar un agroecosistema familiar diversificado de tipo agroecológico, desde un enfoque participativo e integral. En el marco de una Investigación Acción Participativa (IAP), se implementó el método Lume para el estudio de un caso. En la IAP participaron INCUPO, el Grupo Agroecológico Las Tres Colonias y el Consejo de Garantía Participativa (CGP). Se realizó el croquis, la línea de tiempo, el diagrama de flujos y el análisis de los atributos de sustentabilidad. El análisis de la trayectoria permitió identificar momentos claves que determinaron el cambio de la configuración del agroecosistema. El diagrama de flujos reconfirmó a la diversidad como estrategia familiar de comercialización y de autoconsumo. El análisis de atributos sistémicos mostró buenos valores de sustentabilidad $(0,84)$. La evaluación confirmó que una metodología que integre los aspectos socioculturales es más adecuada para los agroecosistemas familiares, y que un abordaje participativo facilita la construcción del conocimiento integrando los conocimientos no académicos a los procesos formales.
\end{abstract}

Palabras clave: agroecología, soberanía alimentaria, feria agroecológica, mercados locales.

\begin{abstract}
In Corrientes center-west area, the expansion of the conventional agricultural model represented a breaking point in production for self-consumption. However, diversified family farmers for selfconsumption and local marketing still persist, such as the farming families producers from The Three Colonies Agroecological Group. These agroecosystems cannot be assessed only through profitability or productivity, since their aims are determined by other elements rather than financial ones. LUME Method of agroecosystems ecological-economic analysis is a suitable proposal to carry out this assessment. The aim of this work was to assess a diversified family agroecological ecosystem from a Participatory Action Research (IAP), thus LUME method was applied in order to study a case. INCUPO, The Three Colonies Agroecological Group and the Participatory Guarantee Council (CGP) participated in the IAP. Then, the sketch, timeline, flow chart and analysis of the sustainability attributes were made. The analysis of track record allowed identifying key moments that determined a turning point in the agroecosystem. The flow chart reassured diversity as a family marketing and self-consumption strategy. The analysis of systemic attributes showed good sustainability values (0.84). The assessment confirmed that a methodology that integrates sociocultural aspects is far more suitable for family agroecosystems, and that a participatory approach fosters the construction of knowledge by integrating non-academic expertise into formal processes.
\end{abstract}

Key Words: agroecology, food sovereignty, agroecological fair, local markets.

Recibido: 09/jun/2020. Aceptado: 01/sep/2020 


\section{INTRODUCCIÓN}

La expansión del modelo de agricultura convencional o moderna, basado en un elevado uso de insumos, permitió un aumento de la productividad y rentabilidad de los sistemas productivos, pero estuvo asociado a grandes impactos sociales, ambientales y económicos (Sarandón y Flores, 2014), desplazando a muchos agricultores de menores recursos que no pudieron adaptarse a las nuevas tecnologías (INTA, 2005).

En el centro-oeste de la provincia de Corrientes, esta expansión representó un quiebre de las producciones para autoconsumo (Valenzuela, 2006) y de la autonomía de los agricultores familiares, ya que se produjo la simplificación de los sistemas que se volcaron únicamente a los cultivos comerciales. A pesar de que más del $75 \%$ de los productores de la región son familiares de pequeña escala (Cad, Lipori, Chifarelli, Pino y Ramilo, 2011), la mayoría producen bajo la lógica de la agricultura moderna. Sin embargo, aún persisten otros en cuyo modelo productivo prevalecen las tecnologías de procesos y la diversificación productiva en función del autoconsumo y la comercialización a escala local, como las familias productoras del Grupo Agroecológico Las Tres Colonias (Pereda, Driutti y Parodi, 2015).

Las familias de este grupo, hace más de 15 años llevan a cabo una experiencia de producción basada en los principios de la Agroecología, entendiendo a la misma como un concepto integral, que abarca más allá de los aspectos ecológico-productivos. Tal como Expresa MAELA (Movimiento Agroecológico de América Latina y el Caribe), "Concebimos a la Agroecología como un sistema de principios, acciones y saberes en el campo político, social, cultural y productivo en el territorio y en las comunidades, que parte de los saberes campesinos e indigenas y genera transformaciones profundas desde los sistemas agroalimentarios, garantizando la Soberanía Alimentaria de los pueblos" (MAELA, 2020). Este modelo de agricultura familiar agroecológica coexiste en la región con los sistemas productivos convencionales.

En los agroecosistemas familiares diversificados, la unidad doméstica está integrada a la unidad productiva, conformando un núcleo social de gestión del agroecosistema (NSGA) (Petersen, Silveira, Bianconi Fernandes y Gomes de Almeida, 2017). En estos sistemas, las estrategias de las familias productoras combinan en sus objetivos la reproducción de las unidades productivas y la satisfacción de las necesidades de consumo de la familia, variando en función del momento del ciclo familiar y de la coyuntura económico-productiva por la que se esté atravesando (Balsa, 2011). Dado que los objetivos de la unidad productiva y la toma de decisiones se rigen mayormente por elementos extraeconómicos, no pueden ser evaluados únicamente a través de la rentabilidad o la productividad, sino que es necesario considerar otros aspectos que visibilicen las distintas racionalidades económicas y ecológicas (Moreira, Nazário, Amâncio, Tavares y Amâncio, 2018).

El método de análisis económico-ecológico de agroecosistemas, conocido como método Lume (Petersen et al., 2017; 2020), fue desarrollado para analizar las estrategias de producción y reproducción en la agricultura familiar, realizando una reflexión conjunta con la familia productora a partir de las formas de manejo del agroecosistema (Petersen et al., 2017). Por ello, constituye una propuesta adecuada para evaluar los múltiples aspectos que intervienen en la toma de decisiones en los sistemas familiares diversificados, a diferencia de un análisis cásico (costo-beneficio) que solo contempla el aspecto económico sin tener en cuenta los objetivos extraeconómicos, la contextualización y la trayectoria histórica. El objetivo del trabajo fue evaluar un agroecosistema familiar diversificado de tipo agroecológico, desde un enfoque participativo e integral, utilizando el método Lume.

\section{METODOLOGÍA Y RESULTADOS OBTENIDOS}

\section{Área de estudio}

El área de estudio es el departamento de Bella Vista, provincia de Corrientes, Argentina. En la región más del $75 \%$ de los productores son familiares de pequeña escala (menos de 25 ha), ocupando menos del $18 \%$ de la superficie (Cad et al., 2011). En los sistemas más comerciales la citricultura, la horticultura y la producción de frutilla, se realizan en monocultivo, con escasa diversificación productiva y un alto uso de plaguicidas para el control de adversidades bióticas. En los sistemas familiares diversificados frecuentemente se produce con bajo uso de insumos, en chacra a campo, en huerta a campo o bajo cubierta, en complemento con animales de granja, ganadería, apicultura, etc. 


\section{Grupo Agroecológico Las Tres Colonias}

El Grupo Agroecológico Las Tres Colonias está integrado por diez familias productoras de las colonias 3 de Abril, Progreso y Desmochado (departamento de Bella Vista). Inició la experiencia de producción agroecológica en el año 1998, con el acompañamiento de INCUPO (Instituto de Cultura Popular). Algunas familias producían de forma natural desde los inicios del grupo, mientras que otras atravesaron por un proceso de transición desde sistemas convencionales hacia sistemas agroecológicos. Cada agroecosistema difiere en cuanto a dotación de recursos y actividades productivas predominantes, siendo la diversificación productiva el denominador común. Son sistemas de pequeña escala (entre 2 y 50 ha), en los que desarrollan variadas actividades: agrícolas (horticultura, frutales, sementera), ganaderas (vacuna, aves y cerdos) y manufactura a escala familiar (harina de maíz, queso, dulces, licores, panificados) (Pereda, 2011; Fleita, 2011). El grupo se formaliza en el año 2006, inaugura la feria agroecológica en el año 2009 y crea el Sistema de Garantía Participativa en Producción Agroecológica (SPG) en el año 2011 (Pereda et al., 2015).

\section{Proceso de Investigación Acción Participativa}

A fines del año 2018, INCUPO inició un proceso de Investigación Acción Participativa (IAP) (Balcázar, 2003; Lacombe, Couix y Hazard, 2018) con el Grupo de Productores Agroecológicos Las Tres Colonias y el Consejo de Garantía Participativa de Productos Agroecológicos de Bella Vista, Corrientes (CGP). Para realizar la IAP se tomó como base el método de análisis económico-ecológico de agroecosistemas (método Lume), desarrollado por AS-PTA Brasil (Petersen et al., 2017; 2020), el cual se conoció a través de un taller organizado por MAELA. El objetivo general de la IAP fue revitalizar el Sistema Participativo de Garantía de Productos Agroecológicos de Bella Vista a partir de la utilización del método. Se trazaron tres objetivos específicos: 1) recuperar la historia del grupo e identificar situaciones claves que sirvan para proyectar líneas de acción en pos de la autonomía, 2) proponer herramientas que permitan profundizar la agroecología en las chacras y en la comunidad, 3) dinamizar el CGP.

En la IAP se propuso implementar el método Lume para analizar tanto al grupo como a cada agroecosistema, desarrollando y aplicando los distintos instrumentos metodológicos de manera grupal. Para ello, se optó por la realización de talleres mensuales con todos los miembros del grupo agroecológico, miembros de INCUPO y miembros del CGP, así como visitas previas a cada taller (Figura 1). Para el análisis de cada agroecosistema a nivel individual, se fueron incorporando progresivamente los instrumentos metodológicos, analizando una familia productora por cada taller. Para ello, cada familia productora fue visitada por INCUPO y el CGP previamente al taller en el que se abordó su caso, para ir acompañando la confección de los instrumentos metodológicos correspondientes.

La IAP aún se encuentra en proceso, finalizando la etapa de análisis cualitativo y comenzando la etapa de análisis cuantitativo. En este trabajo se presentan los resultados del análisis cualitativo de uno de los casos abordados en el proceso de investigación-acción.
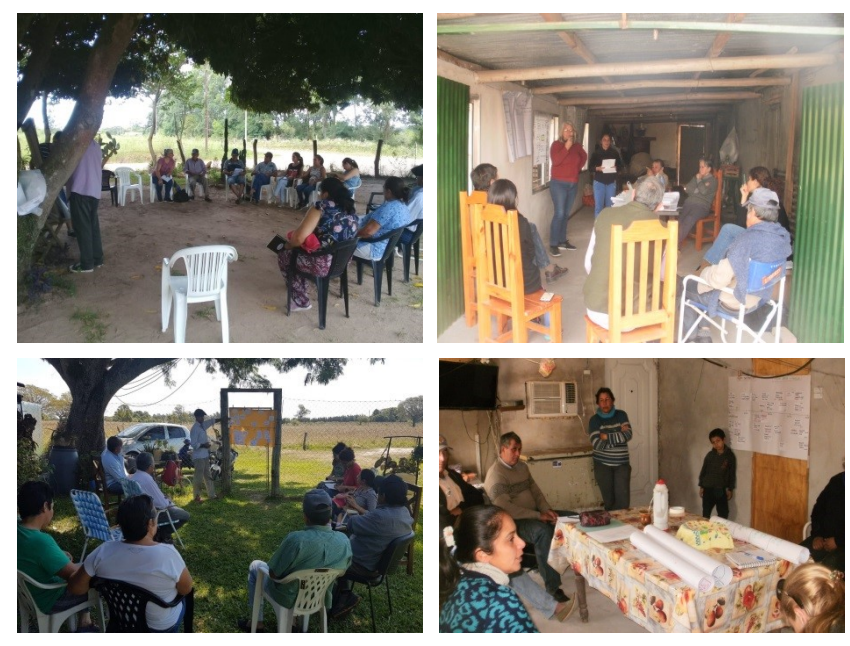

Figura 1. Talleres de implementación del método Lume. Grupo Agroecológico Las Tres Colonias. Bella Vista, Corrientes, Argentina. Fotos: Claudia Toffanelli, Carolina Almada. 


\section{Método Lume}

Este método busca analizar las estrategias de producción y reproducción en la agricultura familiar realizando una reflexión conjunta con la familia productora, orientando la construcción de conocimientos en etapas sucesivas de levantamiento y análisis de información sobre el funcionamiento económico-ecológico de agroecosistemas (Petersen et al., 2017). Está estructurado en dos grandes etapas: etapa de análisis cualitativo (estructura y funcionamiento del agroecosistema) y etapa de análisis cuantitativo (flujos económicoecológicos). La entrevista semiestructurada es el instrumento que se utiliza para el relevamiento de la información del NSGA. Ésta se realiza en dos etapas. En la primera etapa se levanta la información cualitativa sobre la estructura y funcionamiento del agroecosistema. En la segunda etapa se levanta la información cuantitativa sobre los flujos económico-ecológicos.

Para la comprensión de los agroecosistemas y de sus trayectorias históricas, la información relevada en la primera etapa se ordena y analiza utilizando varios instrumentos metodológicos cualitativos:

El croquis: representación visual de las diferentes estructuras y espacios físicos del agroecosistema;

La línea del tiempo: representación de la trayectoria del agroecosistema. Tiene la función de registrar los principales eventos/acontecimientos de la trayectoria del agroecosistema, lo que permite organizar la información significativa sobre la evolución del NSGA para una mejor comprensión de las estrategias reproductivas implementadas.

El diagrama de flujos: esquema simplificado de la estructura y el funcionamiento económico-ecológico del agroecosistema. Consiste en la modelización de los flujos de insumos y productos, rentas monetarias y no monetarias. Permite representar de manera ideal un agroecosistema para comprender su funcionamiento dinámico y analizarlo, facilitando la comprensión de la organización interna del agroecosistema y sus relaciones con el exterior (contexto).

Los atributos sistémicos de sostenibilidad: conjunto integrado de parámetros cualitativos para el análisis de las estrategias de reproducción económico-ecológica puestas en práctica por las familias. Los atributos sistémicos propuestos por el método son: autonomía, resiliencia, integración social, equidad de género/protagonismo de las mujeres, protagonismo de la juventud. Las evaluaciones se expresan en forma de puntuaciones en una escala de 1 a 5 (donde 5 es lo mejor): 1: muy bajo, 2: bajo, 3: medio, 4: alto, 5: muy alto. Para la asignación de puntajes, se utilizan como base los criterios que definen cada parámetro, ya que actúan como guías para la selección, interpretación y traducción de la información.

En la segunda etapa de la entrevista semiestructurada, el relevamiento de nueva de información se centra en la cuantificación de los flujos económico-ecológicos que fueron representados en el diagrama. Los datos se organizan en tablas para facilitar su posterior carga en la hoja de cálculo que los procesará automáticamente, generando un conjunto de indicadores y gráficos sobre el desempeño económico-ecológico del agroecosistema o de los subsistemas.

\section{Breve descripción del caso analizado}

El agroecosistema estudiado se encuentra ubicado en Colonia Progreso, departamento Bella Vista, provincia de Corrientes, Argentina (Figura 2). Consta de 3 ha propias y 10 ha alquiladas que utiliza en conjunto con otras dos familias productoras (para pastoreo) (Figura 3).

El equipo de trabajo está compuesto por Silvia, el marido (Hugo), la hija (Florencia de 13 años) y la abuela (Tita, madre de Silvia). Silvia y Tita dedican tiempo completo al trabajo en el NSGA, estando a cargo de la producción y de los elaborados respectivamente. Florencia aún va a la escuela y dedica medio tiempo al trabajo en el predio. El marido trabaja fuera del predio, en su propio taller mecánico, por lo que participa de las tareas en la medida que el tiempo se lo permite. Principalmente se encarga de las tareas más pesadas (mejora de infraestructura) y el transporte de productos e insumos. Si bien la familia está compuesta por cuatro hijas, tres de ellas viven fuera del predio y no participan del agroecosistema. 


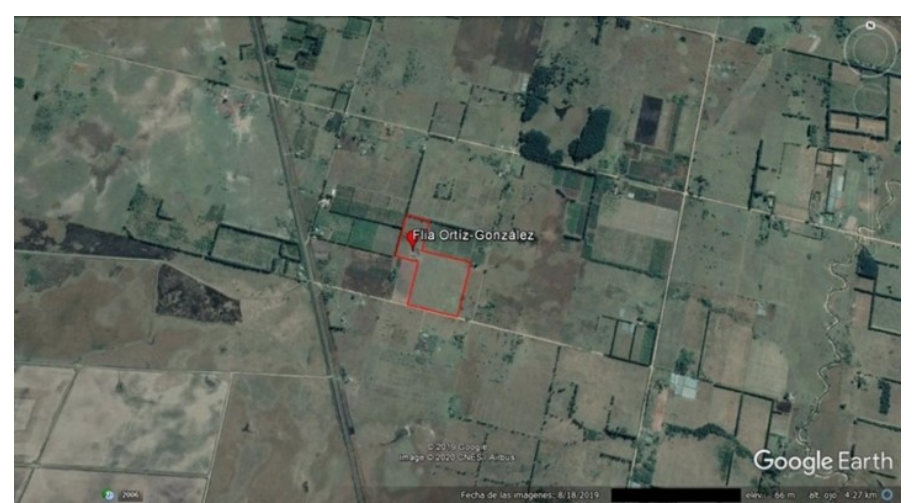

Figura 2. Ubicación del agroecosistema (imágenes extraídas de Google Earth). Familia Ortíz-González. Bella Vista, Corrientes, Argentina.

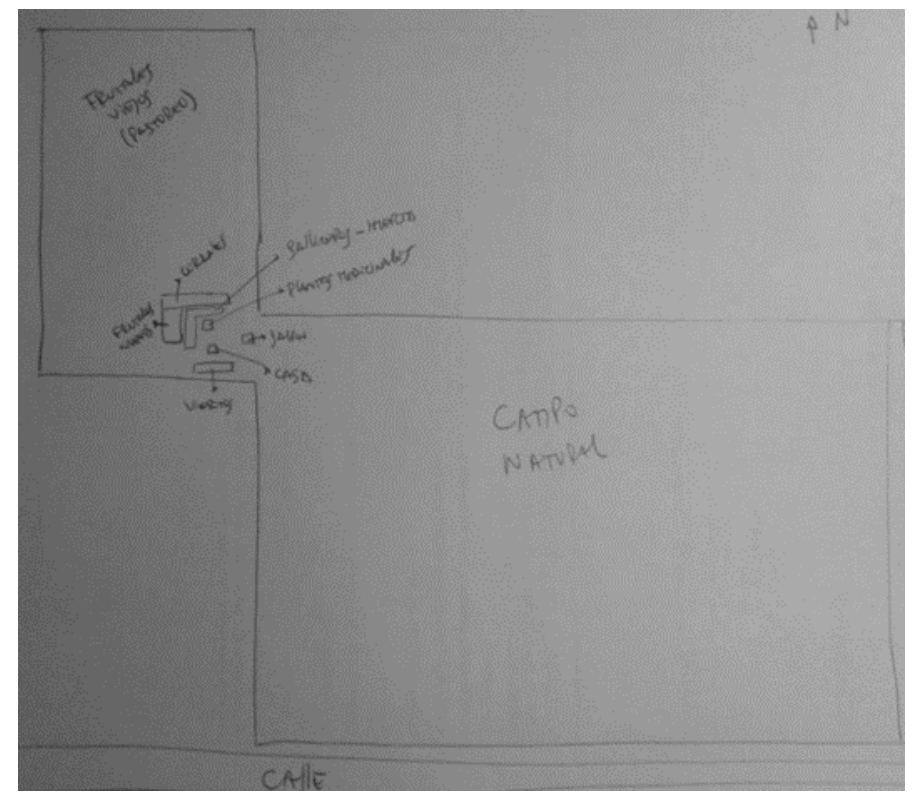

Figura 3. Croquis del agroecosistema. Familia Ortíz-González. Bella Vista, Corrientes, Argentina.

El sistema productivo está orientado principalmente a la cría de gallinas ponedoras, vacas lecheras y plantas ornamentales, aromáticas y medicinales. También tienen frutales y una huerta diversificada para autoconsumo, en la que se asocian distintas producciones: acelga, lechuga, rúcula, espinaca, achicoria, perejil, repollo, cebollita de verdeo, zanahoria, remolacha, romero, orégano, ruda, etc. (Figura 4).

La producción obtenida (verduras, carne, leche, huevos, quesos, dulces, frutas, elaborados, preparados medicinales, hierba seca, etc.), se destina al autoconsumo familiar y la comercialización en venta directa en la feria de productos agroecológicos. La feria es actualmente el único canal de comercialización, porque la producción que tiene sólo alcanza para cubrir ese mercado.

Para la cría de gallinas y producción de huevos tienen diferentes gallineros, organizándolas por grupos. Durante la noche permanecen en los gallineros y durante el día andan libremente. No compran pollitos. Todo lo que tienen fue reproducción propia, de distintas razas que fueron incorporando (criollas -tiki y otras-, negras INTA, camperos, colorada ponedora, parrillero, gallo lego, bataraza). Para la reproducción usa a las tiki para empollar, ya que son muy buenas madres (seleccionan los huevos de otras madres). Todas las gallinas son doble propósito. Lo pollos se engordan y se venden o consumen. Para alimentar a las gallinas compran alimento, principalmente maíz. Para parásitos en las gallinas usa el sauco, que lo pone en el agua una vez al mes.

Las plantas ornamentales, aromáticas y medicinales se producen en "viveritos" que las protegen del clima (acelerando la reproducción) y de las gallinas. No tienen grandes problemas de plagas y nunca tuvieron algún problema que les ocasiono pérdida total. Venden aproximadamente 100 plantas por mes. 

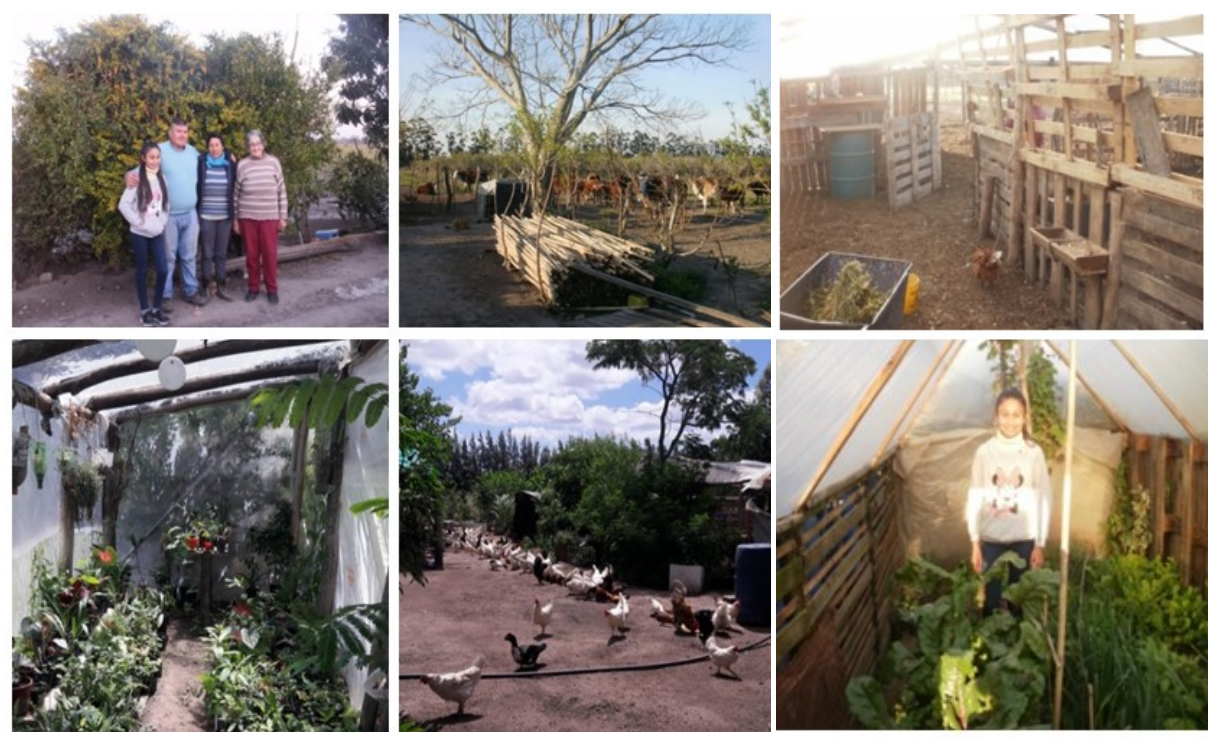

Figura 4. Imágenes del agroecosistema. Familia Ortíz-González. Bella Vista, Corrientes, Argentina. Fotos: Claudia Toffanelli, María José Iermanó.

La producción ganadera se realiza sobre el pastizal, sin pastoreo rotativo, con encierre nocturno. La carga es superior a la que soportaría su sistema, por lo que frecuentemente tienen sobrepastoreo. Cuando realizan el ordeñe les suministran pan para aumentar la ración y aportar energía. Venden las vacas viejas (lecheras) y los terneros. Las vaquillas se las quedan para reposición. En el campo natural (10 ha) y la superficie de quinta fuera de producción ( 2 ha) tienen 15 vacas propias y 13 de otra familia. Aplican las vacunas reglamentarias. Realizan desparasitaciones caseras (parásitos internos y externos).

En la huerta, la preparación del suelo consiste en aplicar estiércol de vaca del propio sistema e incorporarlo manualmente. Se utilizan semillas propias y del programa prohuerta. Preparan sus propios plantines. Para el control de plagas o enfermedades las principales estrategias son la diversificación, uso de trampas, fertilización orgánica, aplicación de preparados caseros de acuerdo a la aparición de adversidades. Tienen frutales que solo usan para consumo propio de fruta y jugo. En cuanto a los recursos forestales, tienen implantada una cortina de eucaliptus (año 2016). En los alrededores de la casa hay numerosos árboles. En el campo natural hay un parche pequeño de monte nativo.

\section{Trayectoria: Línea de tiempo}

El análisis de la línea de tiempo permitió identificar momentos claves para el agroecosistema (Figura 5). La participación en grupos de productores generó nuevas posibilidades de capacitaciones e intercambios, acceso a equipamiento e infraestructura (perforación de agua, alambrados, herramientas, etc.), financiamiento, nuevas formas de comercialización. La participación en el grupo agroecológico también asistió a la mejora en las técnicas productivas, reforzando el esquema productivo original, que desde siempre fue para autoconsumo y de bajo uso de insumos. A partir de la participación en los grupos PSA, el sistema comenzó un cambio importante, ya que el esquema de autoconsumo y venta de cítricos se convirtió, reforzado por la participación en el grupo agroecológico, hacia un sistema de autoconsumo y venta de alimentos agroecológicos en el mercado local.

La activa participación en la feria es uno de los aspectos clave para la actual configuración del sistema productivo, ya que lentamente se fueron especializando en los productos vacantes cuya demanda no estaba cubierta. El otro aspecto tiene que ver con la elección de producciones que se puedan realizar con la mano de obra disponible y las posibilidades físicas de quienes llevan a cabo las tareas. Las hijas mayores se fueron del agroecosistema y no participan de la actividad productiva. Silvia es la única que dedica tiempo completo al trabajo, el resto del equipo participa medio tiempo o menos. Además, se complica la realización de tareas pesadas (como cambiar postes, alambrados, laboreo del suelo, etc.), por lo que Silvia fue optando por actividades productivas que le permitan mínima dependencia de este tipo de tareas. 


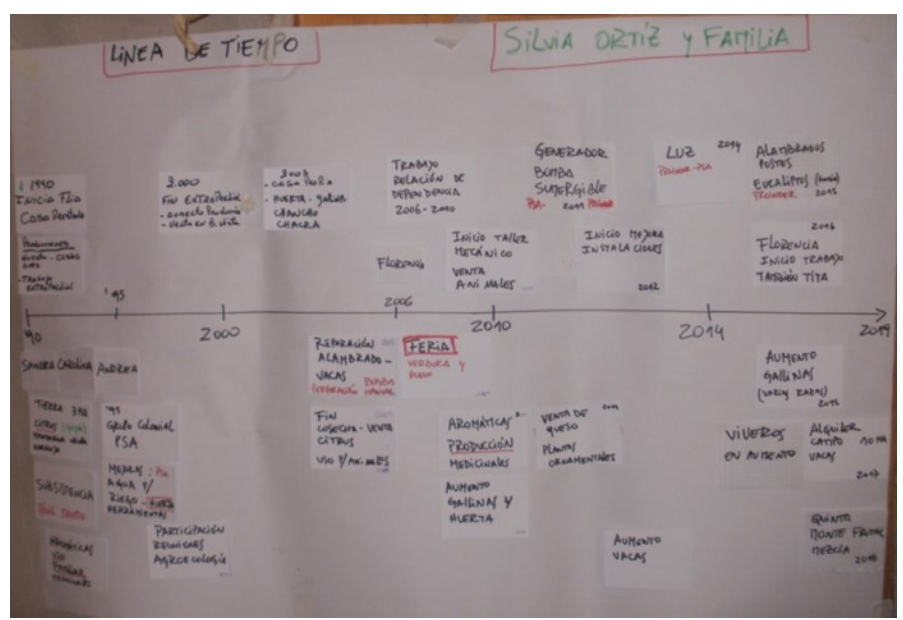

Figura 5. Línea de tiempo del agroecosistema. Familia Ortíz-González. Bella Vista, Corrientes, Argentina.

\section{Diagrama de flujos de insumos y productos}

El diagrama de flujos de insumos y productos se construyó en una visita previa junto con la familia (Figura 6). Se identificaron los siguientes subsistemas de actividades productivas: aves, ornamentales/ medicinales, huerta, vacunos, frutales, elaborados. Se detectaron numerosas interacciones entre los distintos subsistemas. Se observó que los subproductos circulan internamente, funcionando como insumos de otros subsistemas (por ejemplo estiércol para abono, hortalizas y frutas para aves, medicinales para aves, vacunos y huerta), y prácticamente todos los subsistemas suministran productos para autoconsumo de la familia (verduras, carnes, huevo, leche, medicinales) y para los elaborados que se venden luego en la feria. Los corrales, la compostera y la camioneta actúan como mediadores de fertilidad. Un subsistema importante que no figura en el diagrama es el forestal, ya sea por las cortinas de eucaliptus como por el monte nativo que tienen vínculos directos con varios subsistemas, ya que aportan sombra para el ganado, leña para la familia, postes para la infraestructura, entre otras.

En relación al vínculo con el contexto, se observó que la relación con el mercado convencional principalmente tuvo que ver con el consumo de la familia de artículos que no son producidos en el propio sistema y con algunos insumos como combustible, maíz y balanceado para las gallinas, pan, alfalfa y balanceado para las vacas, viruta, macetas para las plantas, alambre tejido, media sombra, etc. La venta de los productos se destina solamente al mercado alternativo. También se observó una fluida relación con la comunidad, dada por el intercambio de productos, plantas, etc., mientras que el estado interviene a través del aporte de semillas, la incorporación de agua y de luz, mejoras, caminos, salud, educación, etc. El diagrama reconfirma a la diversidad como estrategia familiar de comercialización y de autoconsumo.

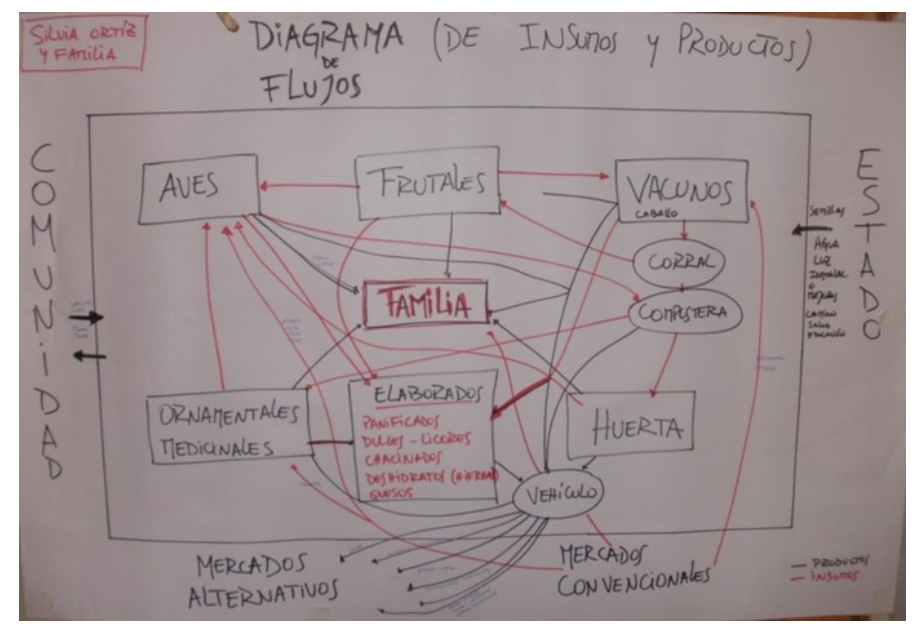

Figura 6. Diagrama de flujos de insumos y productos del agroecosistema. Familia Ortíz-González. Bella Vista, Corrientes, Argentina. 


\section{Análisis de atributos de sustentabilidad}

Se presentan los resultados de los siguientes atributos de sustentabilidad: Autonomía, Resiliencia, Integración social, Equidad de Género/Protagonismo de las mujeres, Protagonismo de los jóvenes (Figura 7). Los parámetros de autonomía tuvieron valores variables, aunque varios de ellos fueron altos a muy altos, tanto en los recursos productivos mercantiles como los autocontrolados. Ello se explica porque el acceso al agua es gratuito y de calidad, se realiza un adecuado manejo de la fertilidad del suelo, la biodiversidad productiva es alta, tanto para garantizar el abastecimiento de autoconsumo familiar como para la venta, las semillas, el material propagativo y las crías son casi completamente propias, el trabajo es completamente familiar. Hubo algunos aspectos débiles relacionados con la tierra y la alimentación animal, que se encuentran relacionados. Aunque una parte de la tierra es de propiedad familiar, la fracción mayoritaria es alquilada con fines de pastoreo. Sin esa tierra, que no es completamente segura en el largo plazo, el sistema debería cambiar su configuración y reducir el número de vacunos. Por otro lado, la producción de aves es casi totalmente dependiente del alimento proveniente del exterior (80\%), mientras que para las vacas deben comprar un $20 \%$ de alimento porque no tienen reservas forrajeras y el campo está saturado. Un aspecto a señalar es que, si bien no compra fertilizante, al adquirir alimento para las aves que luego se convierte en abono para el sistema, indirectamente está incorporando fertilidad. El índice de autonomía fue de 0,84 .

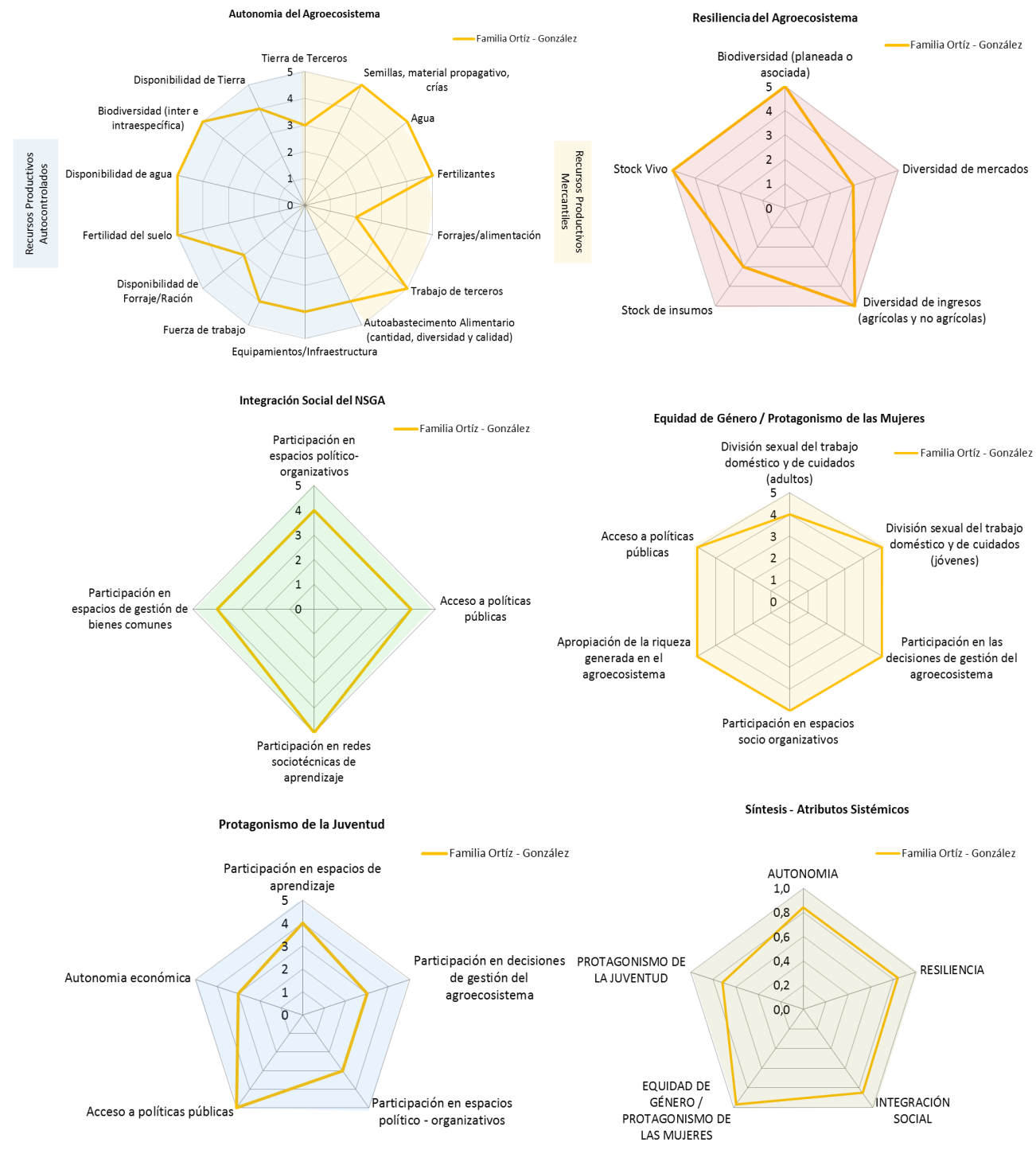

Figura 7. Atributos de sustentabilidad: Autonomía, Resiliencia, Integración social, Equidad de género/ protagonismo de las mujeres y Protagonismo de la juventud, y Síntesis de atributos. Familia Ortíz-González. Bella Vista, Corrientes, Argentina. 
El atributo resiliencia tuvo valores medios y muy altos. Tienen una gran biodiversidad y cuentan con un buen stock de ganado, gallinas y plantas madre. Hubo dos aspectos más débiles: la diversidad de mercados (solo venden en la feria) y el stock de insumos (no cuentan con un stock relevante). El índice de resiliencia fue de 0,84 .

En relación a la integración social, los valores fueron altos a muy altos. La familia participa en distintos espacios organizativos y de alguna manera eso ayuda al acceso a políticas públicas. Además Silvia tiene una participación muy activa en la feria agroecológica. Un aspecto importante a considerar es la capacitación permanente con actividades vinculadas a la producción agroecológica, lo cual ha favorecido la implementación de diversas prácticas que mejoraron su sistema. El índice de integración social fue de 0,85.

En el atributo de Equidad de género/Protagonismo de las mujeres, todos los valores fueron muy altos. La mujer es la que gestiona el agroecosistema y toma la mayoría de las decisiones productivas (el marido trabaja fuera del predio). Hay una tendencia a que las mujeres realicen tareas de todo tipo, tanto productivas como domésticas y de cuidados. En las actividades organizativas la mujer es la más activa, aunque hay un acompañamiento permanente. La apropiación de la riqueza del agroecosistema y el acceso a políticas públicas son equitativos. El índice de Equidad de género/Protagonismo de las mujeres fue de 0,97.

En cuanto al protagonismo de la juventud, los valores fueron principalmente medios y altos. Actualmente solo la hija más chica forma parte del NSGA (13 años). Tiene participación en las tareas del agroecosistema, condicionada por el tiempo de ir a la escuela, y aún no interviene mucho en la toma de decisiones. Si bien no participa de muchas capacitaciones externas, reuniones grupales o feria, tiene una alta capacitación en las tareas productivas, recibida principalmente a través de la familia. El índice protagonismo de la juventud fue de 0,72 .

De acuerdo a los resultados de los índices de atributos sistémicos, el agroecosistema analizado tuvo buenos valores de sustentabilidad. Se destaca la equidad de género con el máximo valor de los atributos evaluados. El valor síntesis de todos los atributos de sustentabilidad analizados fue de 0,84 .

\section{CONSIDERACIONES FINALES}

La información recolectada y analizada, permitió la comprensión de las estrategias llevadas a cabo por la familia productora y la detección de aspectos clave para la configuración del agroecosistema. El análisis de la trayectoria a través de la línea de tiempo fue muy significativo, ya que permitió identificar momentos claves que determinaron el cambio de la configuración del agroecosistema, contribuyendo al discernimiento de las estrategias de reproducción puestas en práctica en el transcurso del tiempo (Petersen et al., 2017). Entre ellos se pueden mencionar el inicio de la participación en grupos de productores, las posibilidades de capacitaciones e intercambios, el acceso a equipamiento e infraestructura, las nuevas formas de comercialización y la especialización en actividades productivas acordes a la mano de obra disponible y las posibilidades físicas de quienes llevan a cabo las tareas. Estos aspectos refuerzan la idea de que los agroecosistemas familiares rigen sus decisiones por parámetros extraeconómicos vinculados a la etapa por la que atraviesa la familia (Balsa, 2011).

La modelización permitió una simplificación de la realidad del sistema para analizar las relaciones involucradas en su funcionamiento dinámico (Moreira et al., 2018). El diagrama de flujos de insumos, productos y mediadores reconfirma a la diversidad como estrategia familiar de comercialización y de autoconsumo. También permitió la detección de numerosas interacciones entre los distintos subsistemas de actividades productivas.

En el análisis de atributos sistémicos, el agroecosistema analizado tuvo buenos valores de sustentabilidad $(0,84)$, destacándose la equidad de género con el máximo valor de los atributos evaluados. Varios aspectos determinan estos buenos valores, entre ellos, el acceso al agua gratuito y de calidad, la alta biodiversidad productiva (tanto para garantizar el abastecimiento de autoconsumo familiar como para la venta), la autoproducción de semillas, material propagativo y crías, el trabajo completamente familiar, la participación en distintos espacios organizativos y la activa participación en la feria agroecológica. 
La evaluación confirmó que una metodología de evaluación que integre los aspectos socioculturales es más adecuada para los agroecosistemas familiares, y que un abordaje participativo facilita la construcción del conocimiento, reintegrando los conocimientos no académicos en los procesos formales de producción de conocimientos sobre realidades agrarias, sistemas agroalimentarios y dinámicas de desarrollo rural.

\section{BIBLIOGRAFÍA}

Balcázar, F.E. (2003). Investigación acción participativa (IAP): aspectos conceptuales y dificultades de implementación. Fundamentos en humanidades, I/II(7-8), 59-77. Recuperado de https://dialnet.unirioja.es/ servlet/articulo?codigo $=1272956$

Balsa, J. (2011). Notas para la caracterización de la agricultura familiar. Actas de las VII Jornadas Interdisciplinarias de Estudios Agrarios y Agroindustriales, Buenos Aires. Recuperado de https:// www.dropbox.com/sh/rzkvlue94le9nkm/AABqRoy2HgFU8fyu64sAUDwEa/La\%20agricultura\% 20familiar\%20capitalizada $\% 20 \mathrm{y} \% 20$ tradicional.\%20Chacareros $\% 20 \mathrm{y} \% 20$ campesinos? $\mathrm{dl}=0$ \&preview $=$ Balsa.doc\&subfolder_nav_tracking $=1$

Cad, M., Lipori, M., Chifarelli, D., y Ramilo, D. Atlas población y agricultura familiar en la Región NEA. Buenos Aires: Ediciones INTA. Recuperado de https://inta.gob.ar/documentos/atlas-poblacion-yagricultura-familiar-en-la-region-nea

Fleita, F. (2011). Sistematización OSALA: Proceso de producción y sistema de garantía participativa de productos agroecológicos “Grupo Las Tres Colonias”. Observatorio de Soberanía Alimentaria y Agroecología. Recuperado de http://www.osala-agroecologia.org/IMG/pdf/ Ficha_Final_Fleita_SG_Productos_Agroecologicos.pdf

INTA (2005). Programa Nacional de Investigación y Desarrollo tecnológico para la pequeña agricultura familiar. Documento Base.

Lacombe, C., Couix, N., y Hazard, L. (2018). Designing agroecological farming systems with farmers: A review. Agricultural systems, 165, 208-220. https://doi.org/10.1016/j.agsy.2018.06.014

MAELA (Movimiento Agroecológico de América Latina y el Caribe). (2020). La Soberania Alimentaria y la Agroecología. Recuperado de https://maela.org/.

Moreira, R., Nazário, R., Amâncio, C., Tavares, P., y Amâncio, R. (2018). A análise econômico-ecológica de um agroecossistema no município de Paraty-RJ como ferramenta de planejamento e apoio à transição agroecológica. Cadernos de Agroecologia, Anais do VI CLAA, X CBA e V SEMDF, 13(1). Recuperado de http://cadernos.aba-agroecologia.org.br/index.php/cadernos/article/view/1665/1079

Pereda, M.M. (2011). Sistema de Garantía Participativa - Bella Vista - Corrientes - Argentina. INCUPOPROINDER. Corrientes. 35p.

Pereda, M.M., Driutti, A., y Parodi, M. (2015). Los SPG como promotores de la agroecología y soberanía alimentaria: la experiencia de Bella Vista, Corrientes, Argentina. Actas del V Congreso Latinoamericano de Agroecologia (SOCLA), La Plata. Recuperado de http://sedici.unlp.edu.ar/ handle/10915/54403

Petersen, P., Silveira, L., Bianconi Fernandes, G., y Gomes de Almeida, S. (2017). Método de Análise Econômico-Ecológica de Agroecossistemas. Rio de Janeiro: AS-PTA. Recuperado de http:// aspta.org.br/files/2017/03/2-livro_METODO-DE-ANALISE-DE-AGROECOSSISTEMAS_web.pdf

Petersen, P., Silveira, L., Bianconi Fernandes, G., y Gomes de Almeida, S. (2020). Lume: a method for the economic-ecological analysis of agroecosystems. Reclaiming Diversity and Citizenship Series. Coventry: Centre for Agroecology, Water and Resilience (CAWR) at Coventry University. Recuperado de https:/www.coventry.ac.uk/globalassets/media/global/08-new-research-section/cawr/coventry-brazilbook-aw3.pdf

Sarandón, S.J. y Flores, C.C. (2014). La insustentabilidad del modelo agrícola actual. En Sarandón S.J. y C.C. Flores (eds.) Agroecología: bases teóricas para el diseño y manejo de agroecosistemas sustentables. La Plata: Edulp. Capítulo 1: 13-41. Recuperado de http://sedici.unlp.edu.ar/handle/10915/37280

Valenzuela, C. (2006). Transformaciones agrarias y desarrollo regional en el nordeste argentino. Una visión geográfica del siglo XX. Buenos Aires: La Colmena. 2006. 